\title{
A Bandwidth Sensitive Distributed Continuous Media File System Using the Fibre Channel Network
}

\author{
Cuneyt Akinlar ${ }^{1}$ and Sarit Mukherjee ${ }^{2}$ \\ 1 Computer Eng. Dept., Anadolu University, Eskisehir, Turkey \\ cakinlar@anadolu.edu.tr \\ 2 Lucent Technologies, Holmdel NJ, USA \\ sarit@lucent.com
}

\begin{abstract}
A recent trend in storage systems design is to move disks to a storage area network for direct client access. While several highly scalable file systems have been built using such direct attached disks, they have all been designed for traditional text-based data, and are not well suited for streaming continuous media, i.e., audio and video files, which are characterized by high volumes of data and require strict timing requirements during storage and retrieval. In this paper, we propose a scalable distributed continuous media file system built using Storage Area Network (SAN)-Attached disks, and describe bandwidth and time sensitive read/write procedures for our file system. We present experimental results on the performance of our Linux-based prototype implementation of the file system and show that the file system can provide strict bandwidth guarantees for continuous media streams.
\end{abstract}

\section{Introduction}

A new trend in storage systems design is to move disks, that traditionally reside behind a centralized server (e.g., Network File System (NFS) [6], Symphony [7, Continuous Media File System (CMFS) 1]), to a storage area network (SAN) for direct client access. While several highly scalable file systems have been built using such direct attached disks (e.g., Global File System (GFS) [8], File systems for NASD 4], xFS 2] among many others), they have all been designed for traditional text-based data (i.e., file systems consisting of many small files), and are not well suited for streaming continuous media files, which are characterized by large volumes of data and stringent bandwidth requirements.

In this paper we propose a scalable distributed continuous media file system based on Storage Area Network (SAN)-Attached disks that can provide strict bandwidth guarantees to open media streams. We briefly describe the general architecture of the file system and discuss different ways to implement this "realtime" sensitivity. We present experimental results on the performance of our Linux-based prototype implementation of the file system using the Fibre Channel 
SAN. Our file system appears as a (Ext2fs) 3] file system to users, and can provide strict bandwidth guarantees for continuous media streams.

\section{Architecture of the Distributed File System}

The architecture of the Fibre Channel Distributed File System (FCDFS) is shown in Figure 1(a). Main components are a file server (FCDFS-Server) and several Storage Area Network (SAN)-Attached disks, which are directly exposed to the clients. While the SAN is used for data transmission between the disks and the clients, a control network connects the clients and the server and is used for exchange of file system meta-data and control messages. Although the figure shows separate logical networks for the SAN and the Control Network, both can coexist on the same physical network depending on the capabilities of the SAN.

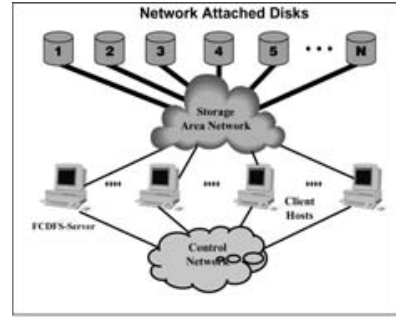

(a)

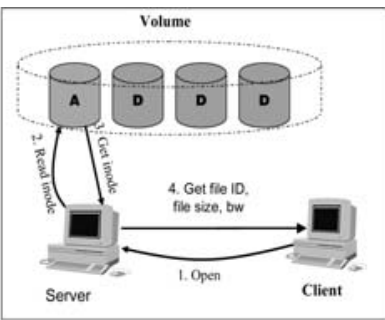

(b)

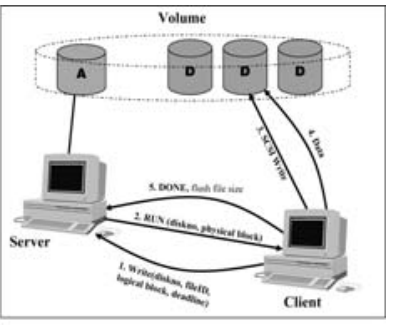

(c)

Fig. 1. (a) Architecture of FCDFS: A file server, client and disks connected together, (b) Open, and (c) Read/Write Operations: A and D denotes attribute and data disks, respectively

FCDFS consists of a client part, which we call the Client-FCDFS, and a FCDFS-Server. While Client-FCDFS is responsible for maintaining open files (streams) and actual reading and writing of the data from/to the disks, the server is responsible for maintaining the meta-data for all volumes, files and directories, and coordinating client access to the data disks. The Client-FCDFS and the server work together in a coordinated fashion to make the operations seamless to the users.

An overview of the file read/write procedure is shown in Figure 1(c). To read or write a block of data, the Client-FCDFS sends a read/write request to the server specifying the logical block of the file to read/write along with the deadline of the request. The server converts the logical block into a (disk, physical block) pair, and schedules the request based on its deadline. Once the client gets a response to its read/write request, it directly accesses the data disk. Therefore, during a read operation, the data directly comes from the disk to the client and during a write operation, the data directly goes from the client to the disk. Excluding the file server from the path of data transfers makes the file 
system scalable. The server is contacted when the file is opened and closed and for read/write request scheduling and acts like a data disk access coordinator. The actual disk request is carried out by the client.

\section{Bandwidth Allocation and Enforcement}

To preserve the quality guarantee across all streams, per stream bandwidth allocation and its enforcement is necessary, and involves the following steps:

1. Bandwidth allocation: Each stream (user) is allocated a certain bandwidth. FCDFS-Client computes a deadline for each pull request using the negotiated bandwidth.

2. Bandwidth enforcement: FCDFS-Server employs a time-sensitive scheduling discipline to ensure that no request misses its deadline.

\subsection{Bandwidth Allocation by Client-FCDFS}

Client-FCDFS keeps per-stream state information, performs bandwidth negotiation during file open, and subsequently assigns a service deadline to each request. The deadline, specified in milliseconds, defines an interval (starting at the current time) by the end of which the request must be completed if the promised stream bandwidth is to be satisfied. FCDFS ensures that the average bandwidth usage per stream is within the negotiated value.

Figure 2(a) shows the procedure used to compute the deadline. To keep track of past bandwidth usage by a stream, the Client-FCDFS keeps a virtual clock [9,

(a)

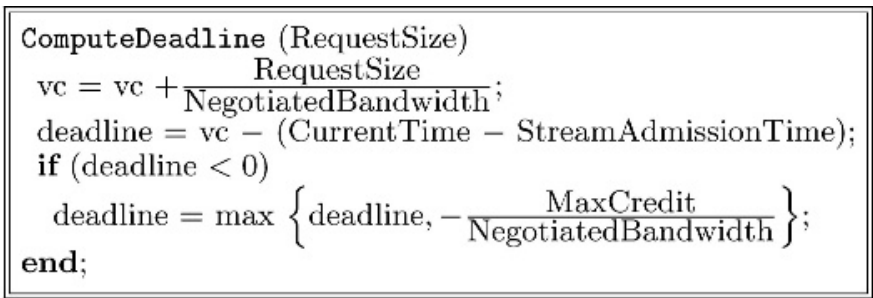

(b)

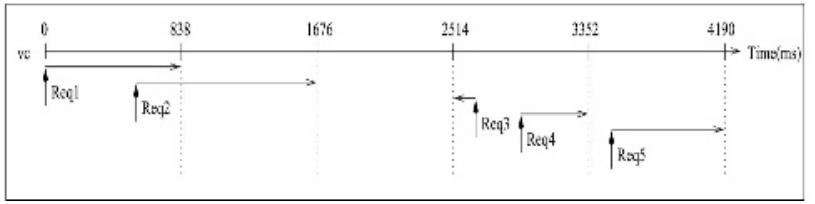

Fig. 2. (a) Algorithm for deadline computation, (b) An example assignment of relative deadlines at the client. Negotiated bandwidth is 10Mbps and requests are in FCDFSblock size of $1 \mathrm{MB}$. Notice that the deadline for the third request is negative, which happens when a stream gets (or asks) less than what it negotiated for a period of time. The negative deadline allows the stream to eventually catch up 
vc, for each stream. During the admission of the stream, vc is initialized to 0 . It is modified only when there is any $\mathrm{I} / \mathrm{O}$ request for the stream. vc keeps account of how long it should have taken for a request to complete had the stream obtained the exact negotiated bandwidth. Note that FCDFS allows the deadline to become negative if a stream does not use its allocated bandwidth. However, FCDFS limits the maximum credit (i.e., the parameter MaxCredit in bytes in the following algorithm) that a stream can accumulate during any inactive period (i.e., no I/O). This ensures that the burst size to the system is limited and regulated.

\subsection{Bandwidth Enforcement by the FCDFS-Server}

The bandwidth enforcement deals with effective and efficient scheduling of the user requests at the server such that a request completes by its deadline. This proceeds in two steps as detailed in the following two sections.

\subsubsection{Global Request Deadline Computation}

Upon reception of a request, the server converts the relative deadline of the request (recall that each request carries a relative deadline that specifies an interval within which the request must be completed) into an absolute deadline so that the relative order among requests from different client hosts can be constructed and maintained. Computation of the absolute deadline of a request, $s^{\text {request }}$, simply is: $s^{\text {request }}=t_{\text {cur }}+r_{\text {deadline }}$, where $t_{c u r}$ is the current time at the server, $r_{\text {deadline }}$ is the relative deadline specified by the client.

\subsubsection{Request Scheduling}

To achieve time-sensitive scheduling of client requests, the server keeps an ordered queue of requests (RQ) for each data disk. The requests in RQ are scheduled using the Earliest Deadline First (EDF) algorithm [5] with the absolute deadline, $s^{\text {request }}$, as the key.

Upon reception of a new request, the server checks the RQ for the disk. If there is no request accessing the disk, then the server schedules the new request by sending a RUN message to the client. Notice that the server schedules a request for execution, but it is the Client-FCDFS that actually executes the request. Thus the server is not on the path of data flow between the disk and the client host.

If there is already a request accessing the disk when a request arrives, the server has two choices: (1) It can wait until the currently executing request completes and then schedule the next request from among the pending requests or (2) If the new request has a smaller deadline, it can preempt the currently executing request and schedule the new one. These two scheduling disciplines are called non-preemptive scheduling and preemptive scheduling.

Figure 3(a) shows how 2 requests from different clients are scheduled by the non-preemptive scheduling discipline. First, Client 1 sends a READ request. Because the disk is idle, the server schedules it immediately, and sends a RUN reply. Client 1 starts accessing the disk. While the disk read is in progress, 


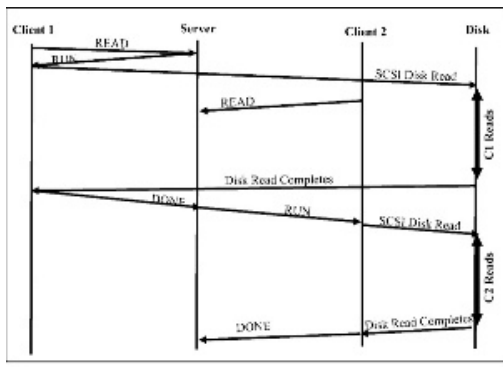

(a)

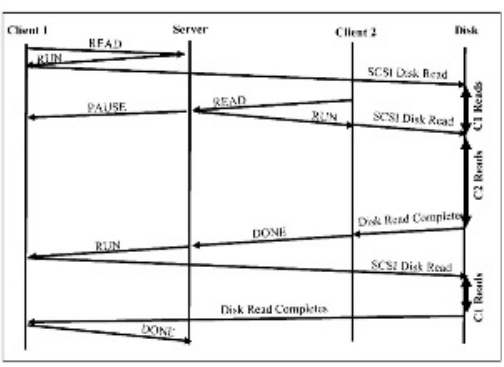

(b)

Fig. 3. (a) Sequence of Events in non-preemtive scheduling, (b) Sequence of Events in preemptive scheduling

Client 2 sends a READ request, which waits its turn until the currently executing request is done. When Client 1 is done reading the disk, it sends a DONE message to the server. The server then schedules Client 2's request and sends a RUN reply. Client 2 reads the data from the disk, and sends a DONE message back to the server when done. If Client 2's request had a stricter deadline than Client 1's request, it might miss its deadline because it must wait until the currently executing request is done.

Figure 3(b) shows how 2 requests from different clients are scheduled by the preemptive scheduling discipline. First, Client 1 sends a READ request. Since the disk is idle, the server schedules it immediately, sends a RUN reply. Client 1 starts accessing the disk. While Client 1 is reading from the disk, Client 2 sends a READ request. Because the request from $\mathrm{Client} 2$ has a stricter deadline than the currently executing request from $\mathrm{Client} 1$, the server preempts it by sending a PAUSE to Client 1 and a RUN to Client 2. From this point on, Client 2 starts reading the disk. When Client 2 is DONE, it sends a DONE message back to the server. The server then reschedules Client 1's request by sending a RUN reply. Client 1 then resumes the execution of the request and finishes it. It sends a DONE message to the server when done.

Since a FCDFS-block resides contiguously on a disk, the non-preemptive policy ensures that disk head movement will be minimal during the service. Therefore, the policy can yield high disk utilization. On the other hand, since servicing a 1MB FCDFS-block may take a considerable amount of time (about $100 \mathrm{~ms}$ for a disk with an average transfer rate of $10 \mathrm{MB} / \mathrm{s}$ ), the higher priority requests may get delayed when the disk is busy, which may cause deadline misses.

\subsubsection{Request Execution at the Client-FCDFS}

Although requests are scheduled by the server, it is the Client-FCDFS that executes them. To execute disk read/write requests, the Client-FCDFS runs a kernel thread for each data disk, which runs the algorithm shown in figure 4 


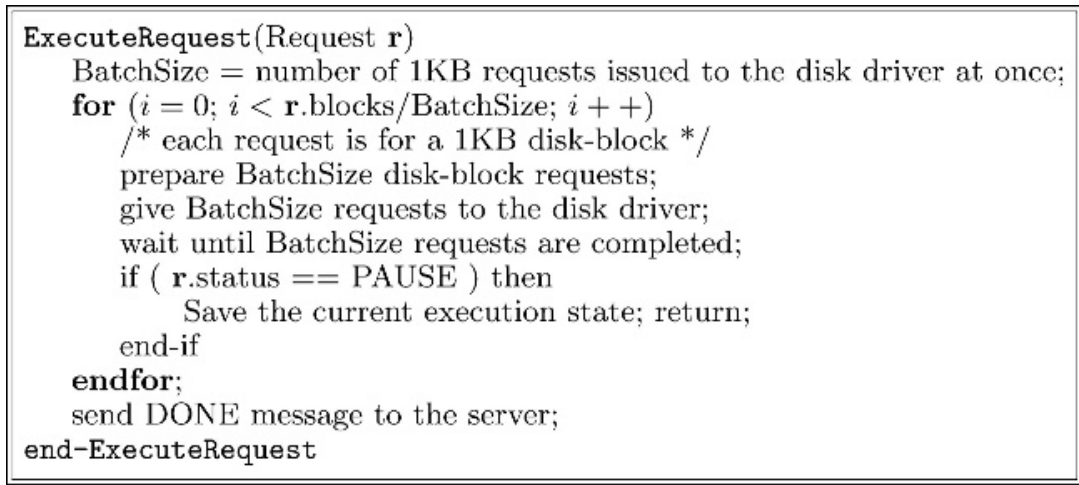

Fig. 4. Request execution at the Client-FCDFS

Instead of issuing a single request for an FCDFS block of size 1MB, ExecuteRequest function issues 1024 read requests, each for a $1 \mathrm{~KB}$ portion of the block. Therefore "r.blocks" in the algorithm is initialized to 1024 and saved properly in the case of a request preemption. ExecuteRequest does not issue each $1 \mathrm{~KB}$ request separately, rather it prepares groups of requests of size BatchSize and gives this group of requests to the disk driver at once. Once BatchSize requests are complete, the next group of requests are prepared. This process continues until the whole block is read/written.

When the server employs the preemptive scheduling discipline, it sends a PAUSE message to preempt a request. Upon reception of a PAUSE message, ExecuteRequest saves the current state of the request and suspends the execution. The execution resumes when the server reschedules the request by sending a RUN message. Notice that the execution of the current request will be preempted only after BatchSize disk requests are complete even if PAUSE message arrives early. Therefore, the value of BatchSize determines how quickly (i.e., the frequency) the Client-FCDFS can respond to preemption messages.

\section{Numerical Results}

To evaluate the bandwidth enforcement policies of our file system, we set up an architecture consisting of 4 client hosts, a server and 8 disks connected to a Fibre Channel Arbitrated Loop (FC_AL) with a maximum transfer rate of $800 \mathrm{Mbps}$. Client hosts and the server are connected with a switched $100 \mathrm{Mbps}$ Ethernet network. The block size for the file system was fixed at $1 \mathrm{MB} 11$. We use a volume with 6 disks, and 18 users equally distributed across 4 client hosts: 2 hosts run 5 and 2 hosts run 4 . We then use the following workloads for evaluation:

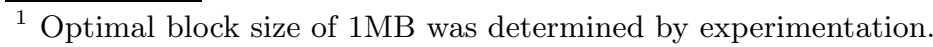




\section{Non-preemptive scheduling Preemptive scheduling}

(a)
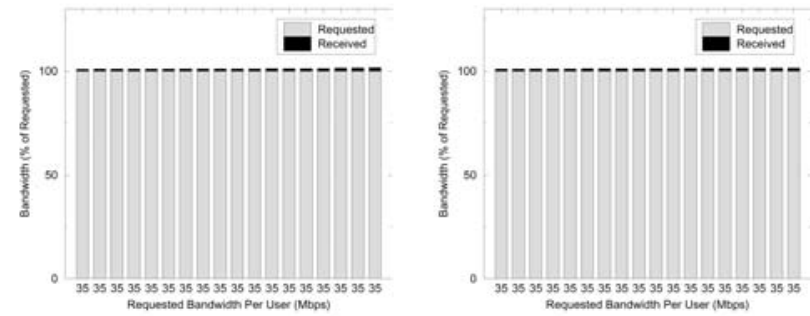

(b)
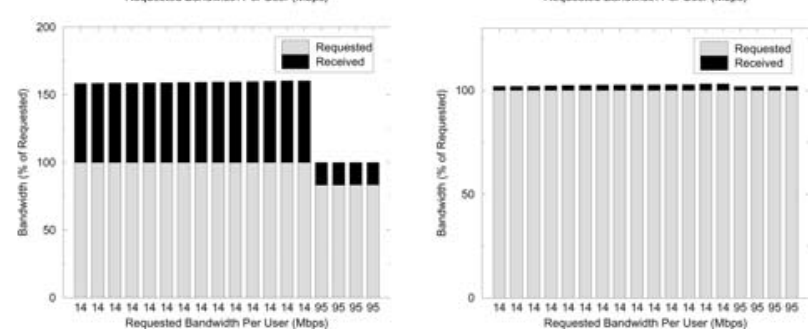

Fig. 5. Workload (a): equal bandwidth distribution, Workload (b): skewed bandwidth distribution. Notice that with non-preemptive scheduling some clients get less than what they negotiated for

(a) Equal bandwidth distribution: All 18 users ask for the same bandwidth of $35 \mathrm{Mbps}$ for a total of $630 \mathrm{Mbps}$.

(b) Skewed bandwidth distribution: 4 very fast users ask for 95Mbps and 14 slow users ask for $14 \mathrm{Mbps}$ for a total of 576Mbps.

Figure 5 shows the results of the experiments. Each user is represented by a vertical bar in the figures. The gray portion of the bar corresponds to the requested bandwidth and is always 100 in the figures. The black portion of the bar shows the percentage of the extra bandwidth received to the requested bandwidth and is simply computed by $\frac{\text { Received-Requested }}{\text { Requested }} \times 100$. The received bandwidth is overlayed on top of the requested bandwidth. If the client gets at least what it requested, the black portion of the bar will be above the requested bandwidth of $100 \%$ and shows the extra bandwidth that the user has received. (This is seen in Figure 5(a)). If however, the user gets less than what it requested, the black portion will be negative and will be below the $100 \%$ requested bandwidth overriding the gray portion of the bar (This is seen in Figure 5 (b)). In the x-axis we show the actual bandwidth requested by the user in Mbps.

First column of Figure 5 (a) and (b) show the results for non-preemptive scheduling: In (a), we see that the requested bandwidths are enforced. Since all users are asking for the same bandwidth, the request deadlines will pretty be similar. So the server will alternate between the requests from the users and non-preemptive EDF scheduling enforces the requested bandwidths for all users. 
In (b) however, we observe that negotiated bandwidths are not enforced: The slow users get a lot more than what they asked for, while the fast users get less than what they asked for.

Observing that non-preemptive EDF scheduling does not always enforce bandwidth, we evaluated the effectiveness of preemptive scheduling by conducting the same set of experiments. We have assumed a BatchSize of 64 in these experiments 2 . The second column of Figure 5 (a) and (b) show the results for each individual user. When all users ask for the same bandwidth, the bandwidths are enforced as in non-preemptive scheduling. When there are a mix of slow and fast users, preemptive scheduling is still able to enforce user bandwidths as shown in Figure 5(b). In this experiment, we observed that $48 \%$ of the requests are preempted by the server, which causes total disk bandwidth to go down to $590 \mathrm{Mbps}$ instead of $640 \mathrm{Mbps}$ with no preemption. That's the price paid to enforce negotiated bandwidths of all users.

\section{Concluding Remarks}

In this paper we presented the architecture and bandwidth enforcement algorithms of our Continuous Media Fibre Channel Distributed File System (FCDFS). Experimental results obtained from our prototype implementation of the file system in Linux platform are presented to evaluate the effectiveness of the file system in enforcing the real-time bandwidth guarantees. We conclude that the proposed file system is well-suited for emerging continuous media applications.

\section{References}

1. D. P. Anderson, Y. Osawa, and R. Govindan. File System for Continuous Media. ACM Transactions on Computer Systems, pages 311-337, November 1992.

2. T. E. Anderson, M. D. Dahlin, J. M. Neefe, D. A. Patterson, D. S. Roselli, and R. Y. Wang. Serverless Network File Systems. ACM Transactions on Computer Systems, February 1996.

3. M. Beck, H. Bohme, M. Dziadzka, U. Kunitz, R. Magnus, and D. Verworner. Linux Kernel Internals. Addison-Wesley, 1998.

4. G. A. Gibson, D. F. Nagle, K. Amiri, J. Butler, F. W. Chang, H. Gobioff, C. Hardin, E. Riedel, D. Rochberg, and J. Zelenka. Filesystems for Network-Attached Secure Disks. Technical Report CMU-CS-97-118, Carnegie Mellon University, July 1997.

5. K. Ramamritham and J. A. Stankovic. Scheduling Algorithms and Operating Systems Support for Real-Time Systems. Proceedings of the IEEE, 82(1), January 1994.

6. R. Sandberg, D. Goldberg, S. Kleiman, D. Walsh, and B. Lyon. Design and Implementation of the Sun Network File System. In Proceedings of the Summer USENIX Conference, pages 119-130, 1985.

\footnotetext{
${ }^{2}$ Optimal BatchSize of 64 was determined by experimentation.
} 
7. P. J. Shenoy, P. Goyal, S. S. Rao, and H. M. Vin. Symphony: An Integrated Multimedia File System. In ACM SIGMETRICS Conference on Modeling and Evaluation of Computer Systems, 1998.

8. S. R. Soltis, G. M. Erickson, K. W. Preslan, M. T. O'Keefe, and T. M. Ruwart. The Global File System: A File System for Shared Disk Storage. Submitted to the IEEE Transactions on Parallel and Distributed Systems, 1997.

9. L. Zhang. Virtual Clock: A New Traffic Control Algorithm for Packet Switching Networks. In Proceedings of SIGCOMM, 1990. 